\title{
Acetylshikonin suppresses invasion of Porphyromonas gingivalis-infected YD10B oral cancer cells by modulating the interleukin-8/matrix metalloproteinase axis
}

\author{
BONG-HAE CHO ${ }^{1,2}$, YUN-HOA JUNG ${ }^{1,2}$, DA JEONG KIM ${ }^{3}$, BOK HEE WOO ${ }^{3}$, \\ JI EUN JUNG ${ }^{3,4}$, JI HYE LEE ${ }^{3,4}$, YOUNG WHAN CHOI ${ }^{5}$ and HAE RYOUN PARK ${ }^{3,4}$ \\ ${ }^{1}$ Department of Oral and Maxillofacial Radiology, School of Dentistry; ${ }^{2}$ Dental Research Institute, \\ Pusan National University Dental Hospital; ${ }^{3}$ Department of Oral Pathology and BK21 PLUS Project, School of Dentistry; \\ ${ }^{4}$ Institute of Translational Dental Sciences, Pusan National University, Yangsan, Gyeongsangnam-do 50612; \\ ${ }^{5}$ Department of Horticultural Bioscience, Pusan National University, Miryang, Gyeongsangnam-do 50463, Republic of Korea
}

Received September 13, 2017; Accepted November 14, 2017

DOI: $10.3892 / \mathrm{mmr} .2017 .8151$

\begin{abstract}
The development of pharmaceutical agents possessing anti-invasive and anti-metastatic abilities, as well as apoptotic activity, is important in decreasing the incidence and recurrence of oral cancer. Cancer cells are known to acquire invasiveness not only through epigenetic changes, but also from inflammatory stimuli within the tumor microenvironment. Accordingly, the identification of agents that can suppress the inflammation-promoted invasiveness of cancer cells may be important in treating cancer and improving the prognosis of patients with cancer. Acetylshikonin, a flavonoid with anti-inflammatory activity, inhibits proliferation and induces apoptosis of oral cancer cells. In the present study, the anti-invasive effect of acetylshikonin on YD10B oral cancer cells infected with Porphyromonas gingivalis, a major pathogen of chronic periodontitis, and the mechanisms involved were investigated. Firstly, we examined whether $P$. gingivalis infection increased the invasiveness of YD10B cells. Results suggested that YD10B oral cancer cells become more aggressive when they are infected with $P$. gingivalis. Secondly, acetylshikonin significantly inhibited the invasion of $P$. gingivalis-infected YD10B cells by suppressing IL-8 release and IL-8-dependent MMP release. These data suggest
\end{abstract}

Correspondence to: Professor Hae Ryoun Park, Department of Oral Pathology and BK21 PLUS Project, School of Dentistry, Pusan National University, 49 Busandaehak-ro, Yangsan-si, Kyeongsangnam-do 50612, Republic of Korea

E-mail:parkhr@pusan.ac.kr

Abbreviations: IL-8, interleukin-8; MMP, matrix metalloproteinase; OSCC, oral squamous cell carcinoma; EMT, epithelial-mesenchymal transition

Key words: Porphyromonas gingivalis, acetylshikonin, invasion, interleukin-8, matrix metalloproteinase that acetylshikonin may be a useful preventive and therapeutic candidate for oral cancer that is chronically infected with periodontal pathogens.

\section{Introduction}

The invasive capability and metastatic potential of cancer cells, as well as their growth rate, are important factors in determining the prognosis of cancer patients. It is well known that the characteristic biological behavior of cancer cells, such as invasion and metastasis, are intrinsically acquired through multiple genetic and epigenetic changes (1). In addition, the importance of extrinsic factors derived from the tumor microenvironment has emerged in recent studies. Among numerous microenvironmental factors, the focus has been on the role of inflammation in carcinogenesis and cancer progression (2-4). It has been suggested that inflammation in the tumor microenvironment contributes to the invasion, metastasis, and resistance to chemotherapeutic agents of various cancers (5-7). The invasive and metastatic characteristics of oral cancer have been major obstacles in the treatment of patients with this disease. However, extrinsic, non-genetic factors that promote invasion and metastasis of oral cancer remain largely unknown while few studies have been conducted on the inflammatory factors involved in acquisition of such pivotal characteristics $(8,9)$. Furthermore, the efficacy of chemotherapeutic agents to modulate the invasiveness of chronic inflammation-induced progression as well as apoptosis of oral cancer cells has been poorly described in an experimental setting.

Periodontitis and oral squamous cell carcinoma (OSCC), one of the most common chronic inflammatory diseases and cancer in the oral cavity, respectively, mostly affect people greater than 40 years of age $(10,11)$. OSCC patients are highly susceptible to chronic periodontitis, suggesting OSCC cells exist in a chronic inflammatory state. However, studies on the effect of periodontitis on the invasiveness and metastatic ability of OSCC are limited. Inflammatory mediators such as IL- 6 and IL- 8 modulate the invasion and metastasis of other types of cancers such as lung adenocarcinoma, as well as breast 
and ovarian cancers (12-14). With multiple bacterial pathogens associated with periodontitis, OSCC patients with this inflammatory disease are continuously exposed to a plethora of periodontopathic bacteria including Porphyromonas gingivalis, Aggregatibacter actinomycetemcomitans, and Prevotella intermedia $(15,16)$. These bacterial pathogens may affect the biological behavior of oral cancer through the modulation of inflammatory mediators and invasion-related molecules. In the present study, we investigated a possible link between chronic periodontitis and the aggressiveness of oral cancer cells by infecting YD10B OSCC cells with $P$. gingivalis, one of the major periodontal pathogens.

Acetylshikonin, a derivative of shikonin isolated from the roots of Lithospermum erythrorhizon, the purple gromwell and Chinese herbal medicine, has been shown to possess anti-inflammatory as well as antibacterial properties (17). Few studies have been undertaken on the effects of acetylshikonin on cancers (18-20). In particular, its role in modulating the biological behavior of cancer cells such as OSCC is largely unknown. In the present study, we examined the possible utility of acetylshikonin, a traditional anti-inflammatory agent, in the suppression of the invasiveness of $P$. gingivalis-infected YD10B OSCC cells. We also examined the potential molecular mechanisms that underlie $P$. gingivalis-induced changes in the invasive characteristics of YD10B cells, including the altered expression of $P$. gingivalis-induced matrix metalloproteinases (MMPs) and a relevant cytokine.

\section{Materials and methods}

Cancer cells and bacterial cultures. The human oral squamous cell carcinoma (OSCC) cell line, YD10B, from Professor JI Yook (College of Dentistry, Yonsei University, South Korea) was used. YD10B OSCC cells were grown in a 3:1 mixture of Dulbecco's Modified Eagle's Medium and Ham's nutrient mixture F12 (Hyclone, Logan, UT, USA) that contained 10\% fetal bovine serum (FBS; Atlas Biologicals, Fort Collins, CO, USA). The cells were maintained at $37^{\circ} \mathrm{C}$ in a $5 \% \mathrm{CO}_{2}$ humidified incubator. The $P$. gingivalis strain 381 were anaerobically cultured in GAM broth (Nissui, Tokyo, Japan) that contained $5 \mathrm{mg} / \mathrm{ml}$ hemin and $5 \mu \mathrm{g} / \mathrm{ml}$ vitamin $\mathrm{K}$.

Infection of OSCC cells with P. gingivalis. YD10B cells were cocultured with live $P$. gingivalis strain 381 at a multiplicity of infection (MOI) of $1: 100$ at $37^{\circ} \mathrm{C}$. After $2 \mathrm{~h}$, the cells were washed with phosphate-buffered saline (PBS) and then cultured in new fresh media until harvest or the next infection. As a control, YD10B cells were subjected to the same media change and PBS wash but without any bacterial infection.

Reagents and chemicals. Human recombinant interleukin-8 (IL-8) was purchased from Peprotech (London, UK). Acetylshikonin was kindly gifted by Prof. Young Whan Choi (Department of Horticultural Bioscience, Pusan National University, South Korea). Acetylshikonin was dissolved in dimethylsulfoxide (DMSO) at a stock concentration of $4 \mathrm{mM}$, stored at $-20^{\circ} \mathrm{C}$, and diluted before use. All chemicals and reagents were purchased from Sigma (St. Louis, MO, USA), unless otherwise specified.
Flow cytometry analysis. Cells were incubated with FITC-conjugated antibodies against CD44 (BD Pharmingen, Franklin Lakes, NJ, USA) and APC-conjugated CD133 (Miltenyi Biotec, Bergisch Gladbach, Germany) for $25 \mathrm{~min}$ at $4^{\circ} \mathrm{C}$ in the dark. The stained cells were immediately analyzed using a flow cytometer (FC500; Beckman-Coulter Cytomics, San Jose, CA, USA) equipped with an argon laser at the excitation wavelength of 488 and $633 \mathrm{~nm}$. The results shown were based on the analysis of 20,000 cells.

Western blot analysis. Cell lysates were analyzed using antibodies against the following molecules: Cytokeratin 13 (BD Biosciences, San Jose, CA, USA); $\alpha$-smooth muscle actin (SMA), twist, and $\beta$-actin (Santa Cruz Biotechnology, Dallas, TX, USA); vimentin and slug (Cell Signaling Technology, Danvers, MA, USA).

In vitro invasion assay. Cells were seeded on the upper sides of 24-well Transwell polycarbonate filters $(8 \mu \mathrm{m}$ pore size, Costar, Cambridge, MA, USA) coated with $40 \mu \mathrm{l}$ of matrigel (BD Biosciences) at a 1:2 dilution in serum-free medium. The upper chambers contained 1\% serum DMEM/F12 medium, whereas the lower wells were filled with medium that contained $10 \%$ FBS. After $30 \mathrm{~h}$, cells on the inside of the inserts were removed with cotton tips, and invading cells on the outside of the inserts were visualized after hematoxylin/eosin staining. The filters were mounted on glass slides, and the number of invading cells were counted using a Photoshop counting program (Adobe Systems, San Jose, CA, USA).

Multiplex bead (Luminex) assay. The concentration of MMP-1, MMP-2, MMP-9 and MMP-10 in supernatants of YD10B OSCC cells were measured using a Milliplex Map Human MMP Magnetic Bead Panel 2 kit (Millipore, Billerica, MA, USA) with a Luminex 200 system (Luminex, Austin, TX, USA). Briefly, beads coupled with anti-MMP-1, MMP-2, MMP-9, and MMP-10 antibodies were diluted in blocking buffer. The standards and samples that contained all MMPs were prepared in blocking buffer and then incubated with a bead solution for $2 \mathrm{~h}$ at room temperature. Each well was supplied with the primary antibody mixture. A streptavidin-phycoerythrin mixture was then added to each well, and the beads were resuspended in PBS. The Luminex 200 platform coupled with BioRad Bio-Plex software (BioRad, Hercules, CA, USA) was used to measure MMP levels.

Gelatin zymography. P. gingivalis-infected or IL-8 treated YD10B cells were incubated in serum-free DMEM/F-12 medium for 24,48 , or $72 \mathrm{~h}$. Conditioned media were collected, and electrophoresed in a 10\% SDS-polyacrylamide gel electrophoresis (PAGE) gel containing $0.2 \%$ gelatin $(\mathrm{w} / \mathrm{v})$ for gelatin zymography. After electrophoresis, the gel was washed twice with a solution containing $2.5 \%(\mathrm{v} / \mathrm{v})$ Triton X-100 for $30 \mathrm{~min}$ at room temperature. Then, the gel was incubated under a shaking condition with a reaction buffer for enzymatic reaction, containing $1 \%$ sodium azide $\left(\mathrm{NaN}_{3}\right), 10 \mathrm{mM}$ calcium chloride $\left(\mathrm{CaCl}_{2}\right)$, and $40 \mathrm{mM}$ Tris-hydrochloride $(\mathrm{pH} 8.0)$, for $16 \mathrm{~h}$ at $37^{\circ} \mathrm{C}$. Finally, the gel was stained with $0.25 \%$ (w/v) Coomassie blue and destained with 5\% acetic acid and 2.5\% methanol at room temperature. 


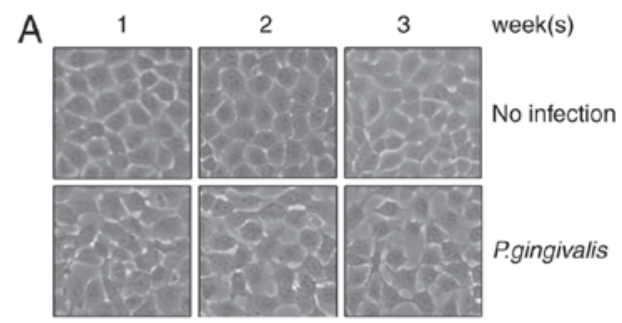

B
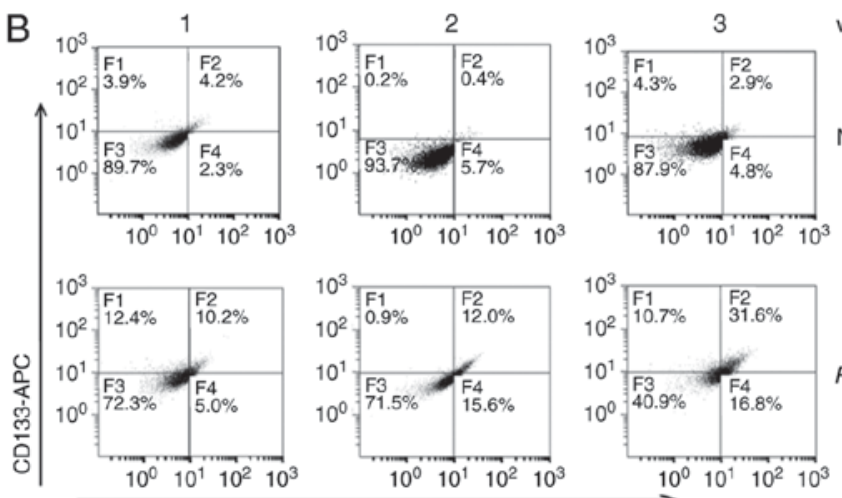

CD44-FITC
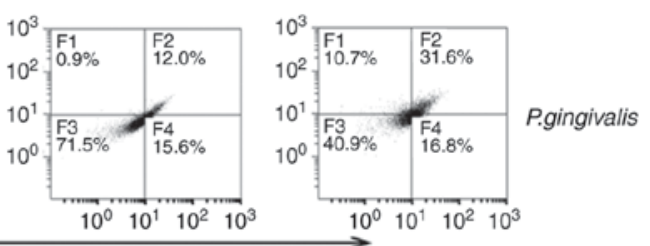

week(s)

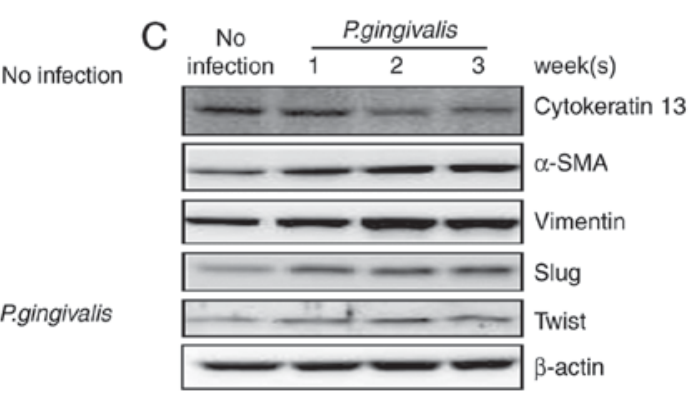

Figure 1. Repeated and prolonged exposure to $P$. gingivalis evoked epithelial-mesenchymal transition (EMT)-like changes in YD10B OSCC cells. Cells were infected with $P$. gingivalis for $2 \mathrm{~h}$ twice a week and grown for 3 weeks. Weekly photographs (magnification, $\mathrm{x} 400$ ) were taken to observe the morphologic changes in $P$. gingivalis-infected OSCC cells compared with non-infected control cells (A). To investigate the appearance of stemness in YD10B cells by repeated $P$. gingivalis infection, expressions of CD44 and CD133 were observed using double immunofluorescence staining (B). Changes in protein expression involved in EMT were analyzed by western blot analysis (C).

IL-8 enzyme linked immunosorbent assay. The conditioned medium of YD10B cells was harvested and analyzed for the presence of IL-8. Enzyme-linked immunoassay (ELISA) $\mathrm{MAX}^{\mathrm{TM}}$ Deluxe sets (Biolegend Inc., San Diego, CA, USA) were used according to the manufacturer's instructions. Briefly, 96-well plates were coated with a primary capture antibody specific for IL-8 in carbonate/bicarbonate buffer overnight. The samples were incubated in plates for $2 \mathrm{~h}$, and a streptavidin-conjugated secondary antibody specific for IL-8 was added. The wells were then incubated with a peroxidase substrate for $30 \mathrm{~min}$, and a $1 \mathrm{M} \mathrm{H}_{2} \mathrm{SO}_{4}$ solution was subsequently added to stop the reaction.

siRNA transfection. IL-8 siRNA (Bioneer Co., Ltd., Daejeon, South Korea) was utilized with the following sequence: CCAAGGGCCAAGAGAAUAUTT. Cells were transfected with $100 \mathrm{nM}$ of IL-8 siRNA or scrambled control siRNA (Bioneer Co., Ltd.) using DharmaFECT transfection reagent (Dharmacon-ThermoScientific, Waltham, MA, USA).

Cell viability assay. The viability of YD10B cells was measured using a 3-(4,5-dimethylthiazol-2-yl)-2,5-diphenyltetrazolium bromide (MTT) assay. Cells were plated in 96-well plates, incubated overnight to reach approximately $80 \%$ confluence, and then treated with varying concentrations of acetylshikonin $(0-2 \mu \mathrm{M})$ for 24 or $48 \mathrm{~h}$. Cells grown in medium containing an equivalent amount of DMSO without acetylshikonin were used as a control. Then, media were removed, and $100 \mu \mathrm{l}$ of MTT $(5 \mathrm{mg} / \mathrm{ml})$ was added to each well. The plates were further incubated for $4 \mathrm{~h}$ at $37^{\circ} \mathrm{C}$. The resulting formazan crystals were solubilized in $200 \mu \mathrm{l}$ of DMSO. The absorbance of colored solutions at $570 \mathrm{~nm}$ was quantified using a PerkinElmer Victor-3 spectrophotometer (Perkin-Elmer, Waltham, MA, USA).

Statistical analysis. Data were analyzed with Student's t-tests for comparisons between two groups. $\mathrm{P}<0.05$ was considered to indicate a statistically significant difference.

\section{Results}

P. gingivalis-infected YD1OB OSCC cells displayed increased invasiveness as well as epithelial-mesenchymal transition-like changes. To verify the invasion of the bacteria into YD10B cells and their subsequent existence within the cells, the presence of $16 \mathrm{~S}$ rRNA from $P$. gingivalis within the cells was analyzed. $P$. gingivalis $16 \mathrm{~S}$ rRNA levels were selectively detected only in $P$. gingivalis-infected cells (data not shown). Our previous study demonstrated that prolonged and repeated infection with $P$. gingivalis twice a week for 5 weeks enhanced the invasiveness of Ca9-22 OSCC cells through the acquisition of cancer stemness as well as epithelial-mesenchymal transition (EMT) characteristics (21), suggesting chronic periodontitis is one of the most important contributing factors in the metastatic progression of oral cancers. In the present study, we observed that sustained short-term infection with $P$. gingivalis induced morphologic changes in YD10B OSCC cells, such as the loss of adhesiveness and a polygonal shape, compared with the absence of morphologic changes in non-infected controls (Fig. 1A). $P$. gingivalis-infected cells also showed increased expression of both CD44 and CD133, representative indicators for cancer stemness (Fig. 1B) $(22,23)$. Additional changes in the expression profile of various EMT markers were detected, 
including up-regulation of $\alpha$-SMA and vimentin, down-regulation of cytokeratin 13, as well as the up-regulation of EMT-related transcriptional factors, such as slug and twist (Fig. 1C). These findings together suggest that $P$. gingivalis, a major pathogen causing chronic periodontitis, can induce a transitional change in YD10B OSCC cells to a mesenchymal phenotype.

P. gingivalis increases expression of matrix metalloproteases in YD1OB OSCC cells. When the invasiveness of $P$. gingivalis-infected YD10 B OSCC cells was examined, OSCC cells chronically infected with $P$. gingivalis exhibited higher invasive properties compared with non-infected cells (Fig. 2A). Matrix metalloproteinases (MMPs) are one of the major effectors in the invasion of cancer cells into neighboring tissues. The enhanced expression of MMPs has been observed in various types of cancer, including colorectal (24), esophageal (25) and lung (26) cancer. We analyzed MMP-1, $-2,-9$ and -10 levels using a multiplex bead assay. Compared with non-infected cells, levels of MMP-1, $-2,-9$, and -10 were substantially increased in a time-dependent manner after $P$. gingivalis infection. The levels of MMPs were maximal at $72 \mathrm{~h}$ post-infection (Fig. 2B). As shown in Fig. 2C, increased expression of MMP-2 and MMP-9 was further confirmed in the supernatant from $P$. gingivalis-infected YD10B OSCC cells using gelatin zymography. These findings suggest that $P$. gingivalis contributes to the increase in invasiveness of YD10B by elevating the level of MMPs. However, further study is needed to define the mechanism that mediates increases of MMPS by $P$. gingivalis.

Increased invasiveness of $P$. gingivalis-infected OSCC cells is modulated by $I L$-8-dependent MMP release. Another important feature of $P$. gingivalis is its ability to secrete extracellular virulence factors, such as fimbriae, endotoxins, and gingipains. These factors stimulate neighboring epithelial cells to produce various cytokines, including interleukins (ILs) and tumor necrosis factor-alpha (TNF- $\alpha$ ), which contribute to inflammatory responses (27-29). Considering the potential link between cancer and bacteria-induced inflammation, the existence of a causal relationship between $P$. gingivalis and oral cancer progression is strongly expected.

Multiple studies have reported that cytokines play a major role in the production of MMPs (30-32). P. gingivalis induces an inflammatory cytokine response in mammalian cells due to its virulence factors. We found that $P$. gingivalis induced the release of IL-8, with a maximal increase observed $72 \mathrm{~h}$ post-infection (Fig. 3A). However, basal levels of other cytokines, such as interferon- $\gamma$, TNF- $\alpha$, RANTES, IL- $1 \beta$, IL- 6 , and IL-10, were very low and not increased by $P$. gingivalis infection (data not shown). Consistent with the increase of MMPs in $P$. gingivalis-infected cells, the conditioned media of YD10B cells treated with $100 \mathrm{nM}$ of recombinant IL-8 treatment contained significantly increased levels of MMPs, in a time-dependent manner, compared to vehicle-treated cells (Fig. 3B). The effects of IL-8 on MMP release and the invasive ability of $P$. gingivalis-infected YD10B cells were further investigated using RNA interference. We confirmed the reduction of IL-8 mRNA and protein by siRNA using real-time PCR and ELISA, respectively (data not shown).
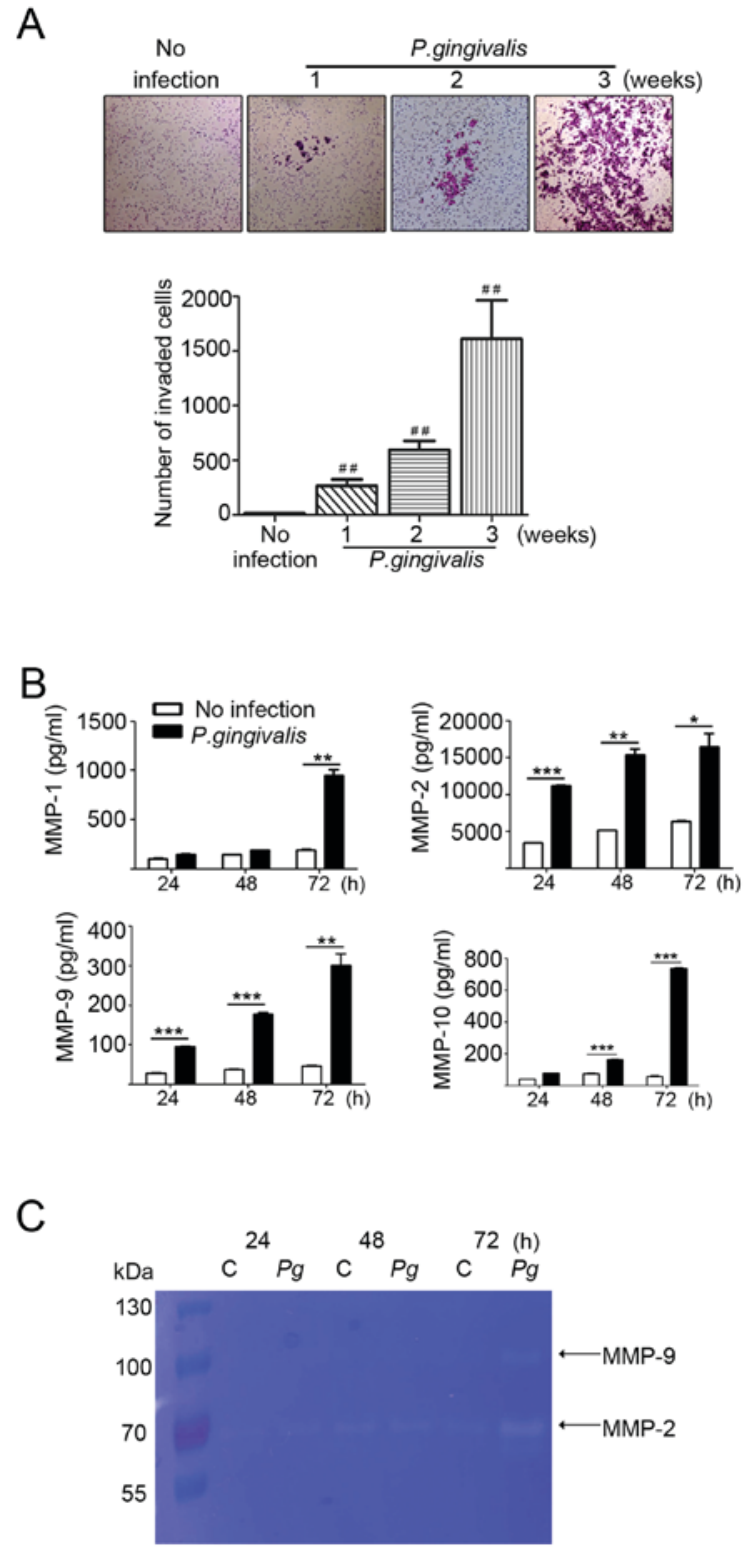

Figure 2. P. gingivalis-infected oral cancer cells display increased invasive ability by modulating MMPs. (A) The invasive ability of YD10B cells that were repeatedly infected with $P$. gingivalis twice a week was examined in an in vitro invasion assay (magnification, X100). (B) OSCC cells were seeded and grown with or without $P$. gingivalis at the indicated time points from 24 to $72 \mathrm{~h}$. The condtioned media of $P$. gingivalis-infected or non-infected cells were harvested, and MMP levels measured using a multiplex bead assay. MMP activity in cell supernatants were analyzed using a zymogram assay (C). All data represent at least three independent experiments. Each bar represents the mean \pm standard deviation. Statistical significance was assessed by paired t test. ${ }^{\#} \mathrm{P}<0.01$, compared with no infection group; ${ }^{*} \mathrm{P}<0.05,{ }^{* *} \mathrm{P}<0.01,{ }^{* * *} \mathrm{P}<0.001$, compared with no infection group as indicated.

Knockdown of IL-8 substantially decreased MMP-1, -2, and -10 release $72 \mathrm{~h}$ post-infection, but a significant change in the MMP-9 level was not noted (Fig. 4). We also observed significant suppression of the invasive ability of $P$. gingivalis-infected YD10B cells following an siRNA approach against IL-8, as well as after treatment with SB2250025, an inhibitor of the IL-8 receptor. In contrast, IL-8 treatment substantially enhanced the invasiveness of YD10B cells compared with vehicle-treated control cells (Fig. 5). These findings strongly support the notion that the IL-8-induced 
A

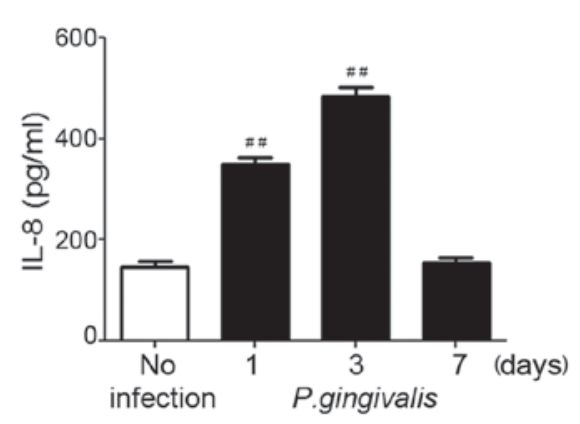

B
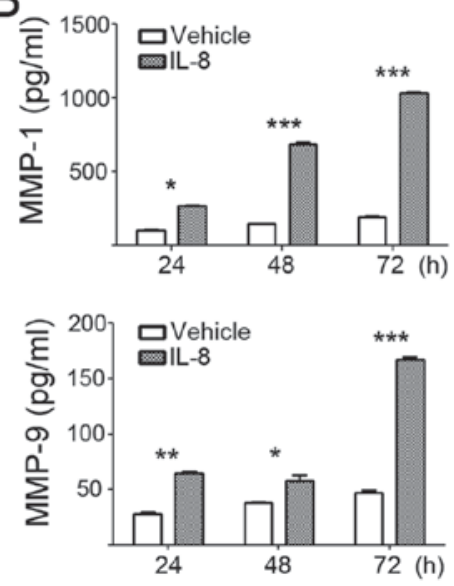
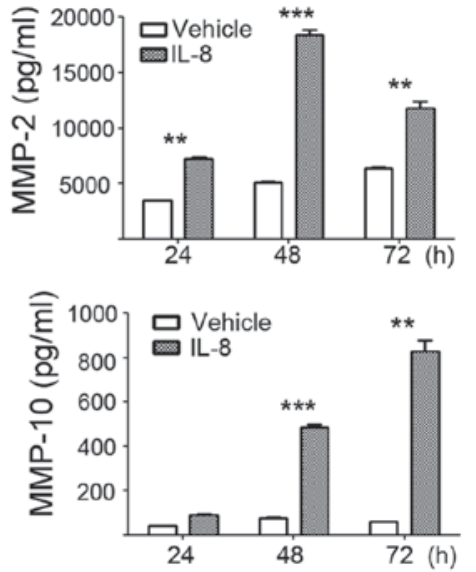

Figure 3. IL-8 significantly increased release of MMPs in YD10B OSCC cells. (A) Cell supernatants of YD10B OSCC cells were harvested for the indicated time points following $P$. gingivalis infection and the level of IL-8 in the supernatants was analyzed using an enzyme-linked immunosorbent assay (ELISA). (B) Cells were treated with IL- 8 for 24,48 , or $72 \mathrm{~h}$, and the amount of matrix metalloproteinases (MMPs) in the supernatant was subsequently determined using a multiplex assay. Statistical significance was assessed by paired t test. ${ }^{\# \#} \mathrm{P}<0.01$, compared with no infection group; ${ }^{*} \mathrm{P}<0.05,{ }^{* *} \mathrm{P}<0.01$, and ${ }^{* * *} \mathrm{P}<0.001$, compared with vehicle-treated group as indicated.

A

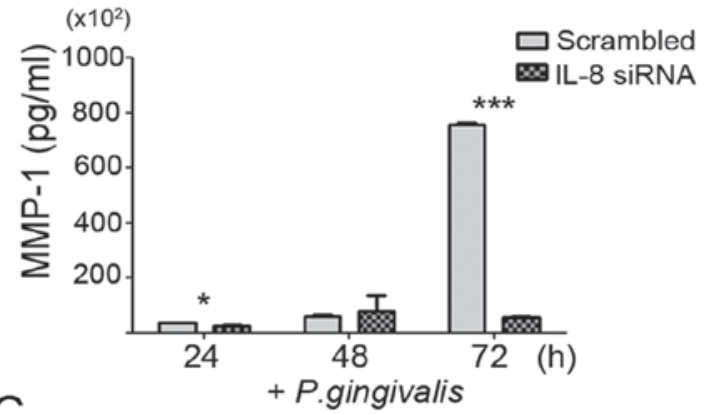

C

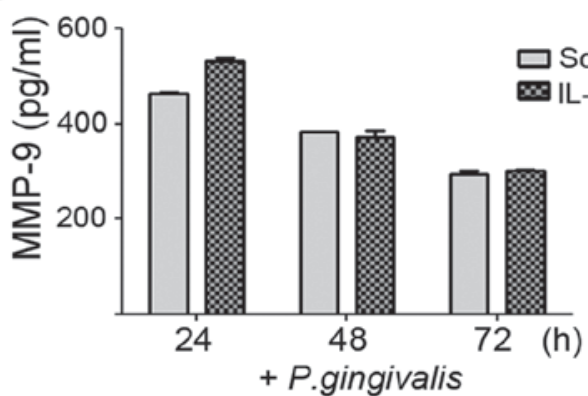

B
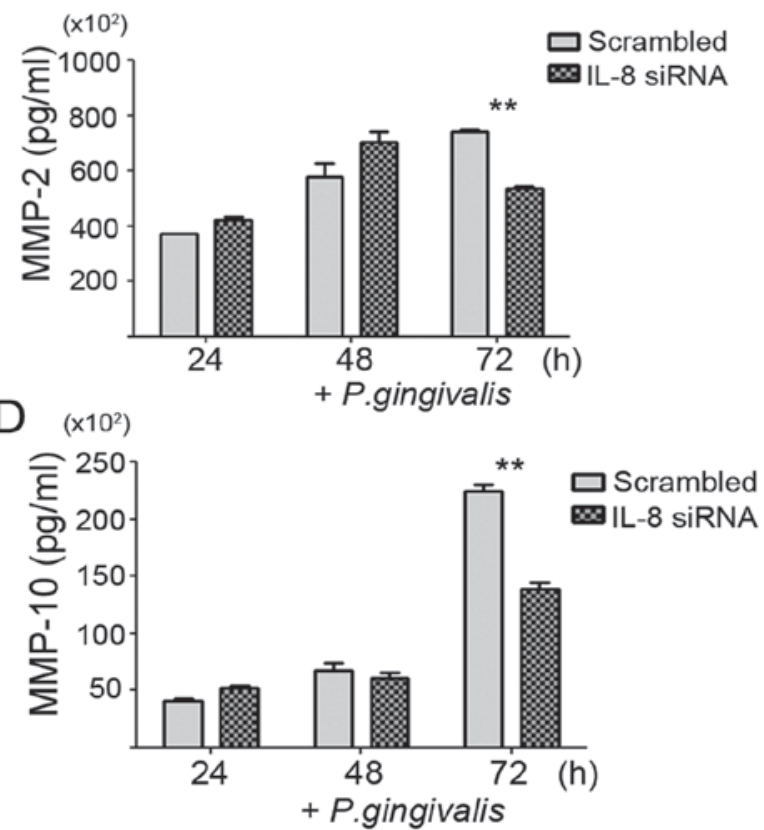

Figure 4. MMPs release induced by $P$. gingivalis is dependent on IL-8. (A-D) To confirm the effect of IL-8 on MMP release, IL-8 expression in $P$. gingivalis-infected OSCC cells was knocked down using an siRNA construct against IL-8, and MMP levels in cells were measured using a multiplex assay. Statistical significance was assessed by paired t test. ${ }^{*} \mathrm{P}<0.05,{ }^{* * *} \mathrm{P}<0.01,{ }^{* * * *} \mathrm{P}<0.00$ as indicated.

upregulation of MMPs in $P$. gingivalis-infected YB10B cells underlies the increased invasiveness of YD10B OSCC cells.

Acetylshikonin inhibits the invasion of P. gingivalis-infected OSCC cells by decreasing release of both $I L-8$ and MMPS. Prior to observing the influence of acetylshikonin on the invasive ability of $P$. gingivalis-infected YD10B OSCC cells, we firstly investigated the toxicity of acetylshikonin to YD10B cells using an MTT assay. Cells were exposed to various concentrations ( 0 to $2 \mu \mathrm{M}$ ) of acetylshikonin for 24 or $48 \mathrm{~h}$ (Fig. 6A). Since acetylshikonin up to a $1 \mu \mathrm{M}$ concentration showed no toxicity to cells, a $0.5 \mu \mathrm{M}$ concentration was used in subsequent experiments. Acetylshikonin substantially suppressed the IL- 8 release that was induced by $P$. gingivalis infection (Fig. 6B). MMP-1, -2, -9, and -10 were significantly reduced with $0.5 \mu \mathrm{M}$ of acetylshikonin treatment in $P$. gingivalis-infected YD10B cells. The suppressive effect of acetylshikonin on MMP release was more potent than the IL-8 receptor inhibitor, SB225002 (Fig. 7). In addition, acetylshikonin of the same concentration potently suppressed the increased invasive ability of OSCC cells induced by $P$. gingivalis infection (Fig. 8). 
A

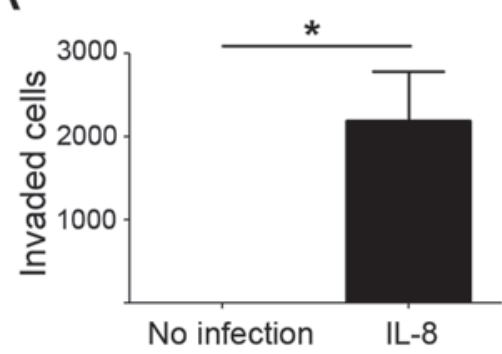

$\mathrm{B}$

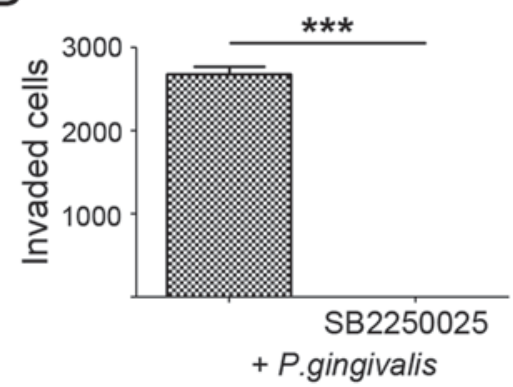

C

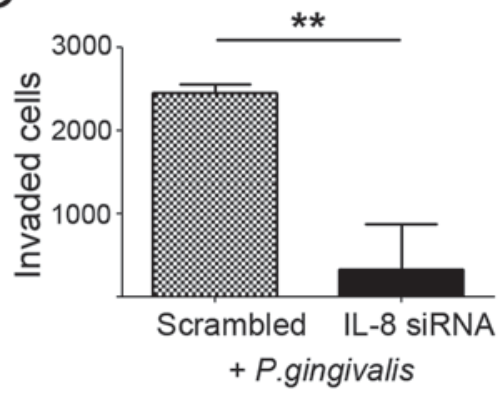

Figure 5. Invasiveness of $P$. gingivalis-infected YD10B OSCC cells is modulated by IL-8. To confirm the effect of IL-8 on the invasiveness of OSCC cells, the invasiveness of YD10B OSCC cells through matrigel-coated transwell membranes by $100 \mathrm{ng} / \mathrm{ml} \mathrm{rIL}-8$ treatment was compared with non-treated OSCC cells (A). In addition, the invasive abilities of SB225002 or IL-8 siRNA treated cells were examined $30 \mathrm{~h}$ after seeding (B and C). Magnification, x40. Statistical significance was assessed by paired $\mathrm{t}$ test. ${ }^{*} \mathrm{P}<0.05,{ }^{* *} \mathrm{P}<0.01,{ }^{* * *} \mathrm{P}<0.001$ as indicated.

A

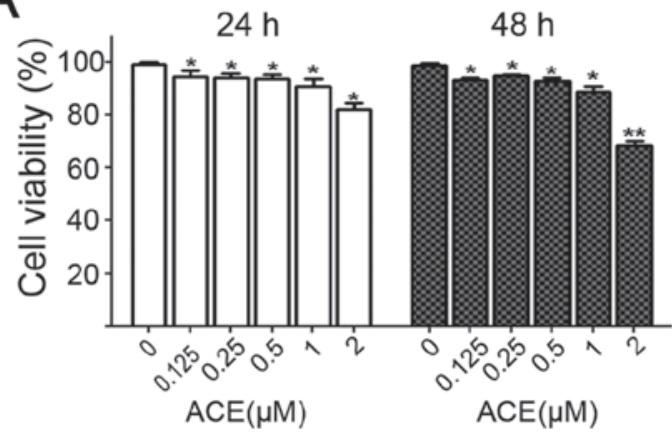

B

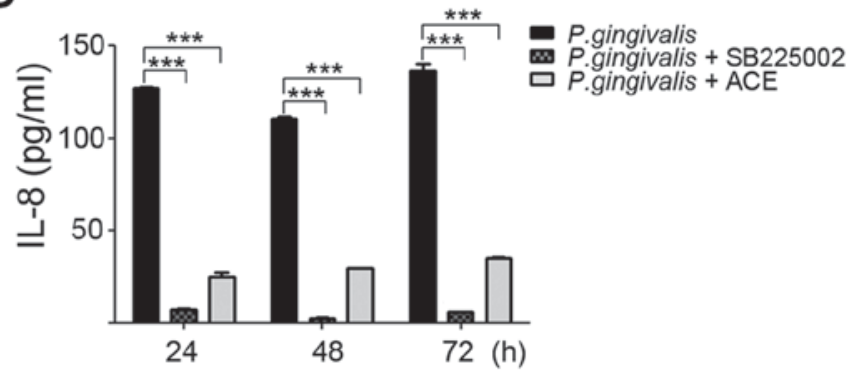

Figure 6. Acetylshikonin inhibited secretion of IL-8 from P. gingivalis-infected OSCC cells. (A) Cells were grown in 96-well plates and treated with 0 , $0.125,0.25,0.5,1$ or $2 \mu \mathrm{M}$ of acetylshikonin for 24 or $48 \mathrm{~h}$. Dimethyl sulfoxide (DMSO) was used as a vehicle control. Cell viability was measured using a 3-(4,5-dimethylthiazol-2-yl)-2,5-diphenyltetrazolium bromide (MTT) assay. (B) P. gingivalis-infected cells were treated with $100 \mathrm{nM}$ of SB225002, an IL-8 receptor inhibitor, or $0.5 \mu \mathrm{M}$ of acetylshikonin for 24,48 , or $72 \mathrm{~h}$. The amount of IL-8 in the supernatant was subsequently determined using ELISA. Statistical significance was assessed by paired t test. ${ }^{*} \mathrm{P}<0.05,{ }^{* * *} \mathrm{P}<0.01$, compared with vehicle-treated control cells; ${ }^{* * * *} \mathrm{P}<0.001$, compared with $P$. gingivalis-infection group as indicated. 1. ACE, acetylshikonin.

A

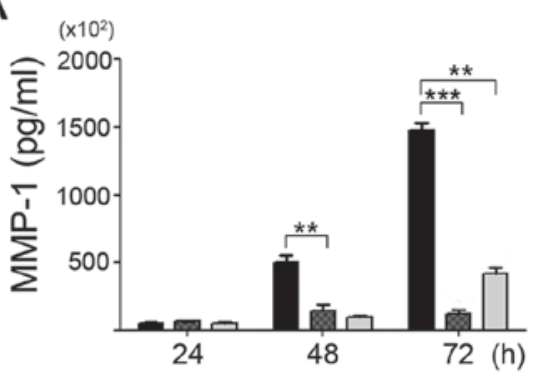

C

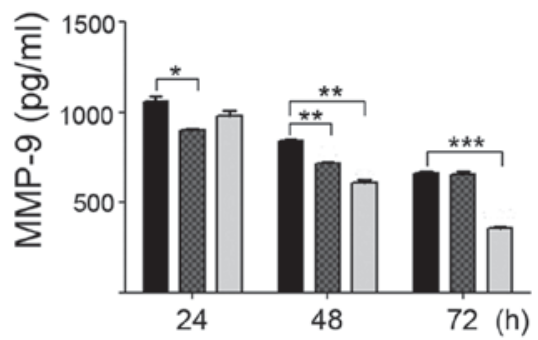

B

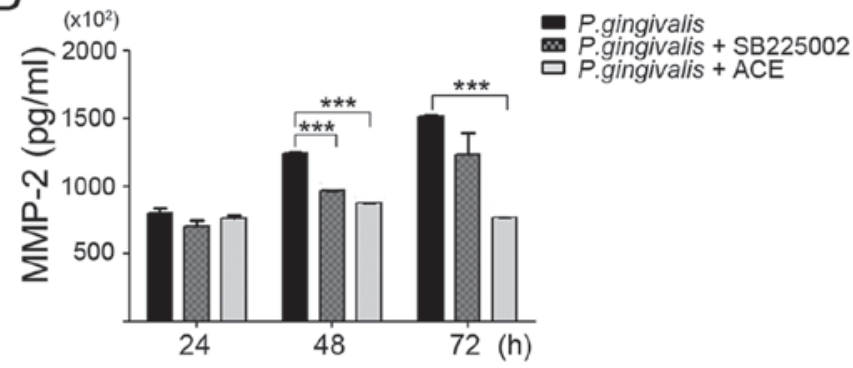

D

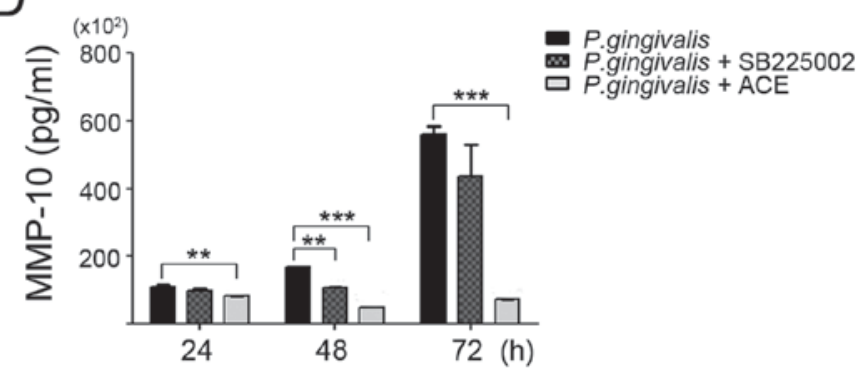

Figure 7. Acetylshikonin significantly decreased release of MMPs in P. gingivalis-infected OSCC cells. (A-D) P. gingivalis-infected cells were treated with $100 \mathrm{nM}$ of SB225002 or $0.5 \mu \mathrm{M}$ of acetylshikonin for 24,48 , or $72 \mathrm{~h}$. The amount of MMPs in the supernatant was subsequently determined using multiplex assays. Statistical significance was assessed by paired t test. ${ }^{*} \mathrm{P}<0.05,{ }^{* *} \mathrm{P}<0.01,{ }^{* * *} \mathrm{P}<0.001$, compared with $P$. gingivalis-infection group as indicated. ACE, acetylshikonin. 


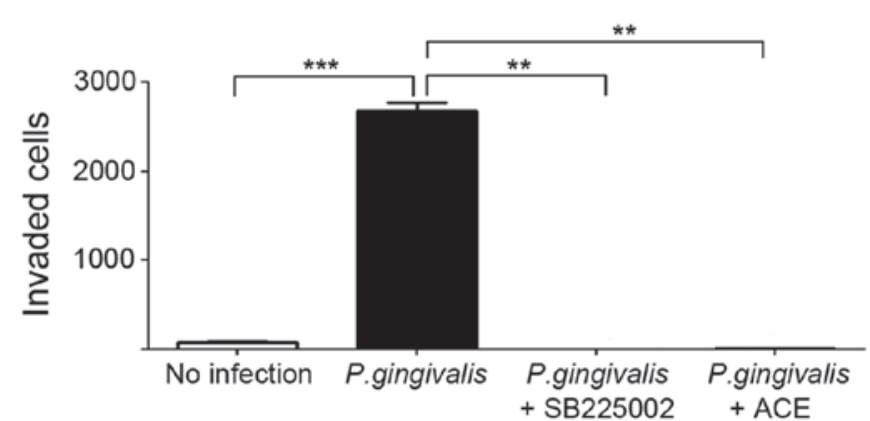

Figure 8. Acetylshikonin effectively suppressed the enhanced invasiveness of $P$. gingivalis-infected OSCC cells. $P$. gingivalis-infected cells were treated with vehicle, $100 \mathrm{nM}$ of SB225002, an IL-8 receptor inhibitor, or $0.5 \mu \mathrm{M}$ of acetylshikonin. The invasiveness of the cells was compared using Transwell assay. Statistical significance was assessed by paired t test. ${ }^{*} * * \mathrm{P}<0.001$, compared with no infection group; ${ }^{* *} \mathrm{P}<0.01$, compared with $P$. gingivalis-infected cells. ACE, acetylshikonin.

\section{Discussion}

While previous studies have focused on the role of pathogens in carcinogenesis, more recent studies have described the effect of microbial infection on the biologic behavior of cancer cells. A number of studies have demonstrated that microbial pathogens, including Epstein-Barr virus, hepatitis $\mathrm{B}$ and $\mathrm{C}$ virus, and Citrobacter rodentium, contribute to the transition of cancers into a more aggressive form, and that they may play a role in the induction of EMT in cancer cells associated with tumor invasion and metastasis (33-36). From recent studies on periodontal pathogens, a link between periodontal pathogens and EMT in oral cancer cells has been suggested $(21,37)$. In the present study, $P$. gingivalis-infected YD10B OSCC cells displayed a morphologic transformation into a mesenchymal cell-like shape. In addition, additional characteristics of EMT have been detected, including epithelial marker repression, and the upregulation of mesenchymal markers as well as the stem cell markers, CD44 and CD133. While the interpretation of this result may be restricted to a single cell line used, these findings are consistent with our previous studies demonstrating an effect of $P$. gingivalis on Ca9-22, another OSCC cell line (21). Regardless of OSCC cell lines used, $P$. gingivalis-infected OSCC cells in both studies exhibited enhanced invasive properties, characteristic of EMT. All these findings strongly support the idea that $P$. gingivalis may induce EMT in the progression of oral cancer.

$P$. gingivalis can induce cytokine production in not only immune cells but also in cells from periodontal tissue $(27,29)$. Previous reports have demonstrated that $P$. gingivalis-infected oral cancer cells secrete cytokines and chemokines, especially IL-6 and IL-8 $(21,38)$. Few studies exist describing the effect of IL- 8 on the invasion and metastasis of cancer cells while cytokine studies have mainly focused on IL-6 $(9,12-14)$. Recently, a role for IL-8 in the migratory and invasive abilities of human ovarian and breast cancer cells has been shown (39). The mechanism involved in the IL-8 promoted invasion of cancer cells has been further clarified by a study showing that invasion and metastasis of bladder cancer can be increased by an IL-8/MMP axis (40). MMPs are known as important molecules in the process of periodontal tissue destruction, as well as in tumor invasion, by playing a key role in the degradation of extracellular matrix proteins. The current finding of increased MMP-1, -2, -9, and -10 in $P$. gingivalis-infected YD10B OSCC cells is in line with our previous reports demonstrating the activation of similar sets of MMPs in other OSCC cell lines $(21,38)$. Our results also indicate the IL-8-induced enhancement of MMPs as a common molecular pathway.

Considering that IL-8 is one of the most important contributors to the enhancement of aggressive behaviors by $P$. gingivalis-infected oral cancer cells, we postulate that anti-inflammatory agents capable of modulating the IL-8 pathway can effectively suppress the invasion of OSCC cells surrounded by microbial pathogens. Recently, natural compounds such as flavonoids have become the focus of attention as anticancer agents and/or adjuncts to chemotherapeutic reagents, although the emphasis is as their utilization as chemopreventive agents rather than for therapeutic benefit $(41,42)$. Of numerous flavonoids, we demonstrated, in a previous study, that acetylshikonin, a flavonoid with an anti-inflammatory potential, has anti-tumor properties after inducing apoptotic cell death on Ca9-22 OSCC cells at a low concentration $(1 \mu \mathrm{M})$, with little to no toxicity to normal cells (18). However, YD10B OSCC cells in the present study were little damaged by acetylshikonin at low concentrations. Instead, herein, we demonstrated that acetylshikonin significantly suppressed IL-8 induction and MMP release as well as the invasive ability of $P$. gingivalis-infected YD10B OSCC cells, with no direct toxic effect. These data suggest that acetylshikonin may be a good candidate as an adjuvant chemotherapeutic reagent that suppresses the invasion and metastasis of oral cancer cells, especially against OSCC cells chronically infected with periodontal pathogens that otherwise have the potential to be transformed into more aggressive populations. Further in vitro and in vivo studies are required to clarify the suppressive role of acetylshikonin and to broaden our understanding of relevant molecular pathways in aggressive $P$. gingivalis-infected oral cancer cells.

In summary, we present data to further confirm the hypothesis that $P$. gingivalis, a major pathogen responsible for chronic periodontitis, induces the development of aggressive behaviors in host YD10B oral cancer cells via the IL-8 dependent release of MMPs. More importantly, acetylshikonin, a flavonoid with an anti-inflammatory potential, effectively prevented $P$. gingivalis-infected YD10B OSCC cells from transforming into more aggressive populations, presumably via the suppression of IL-8 and MMP release. Our results may help to provide a useful platform for developing pharmaceutical adjuvants that will aid traditional therapeutic agents in curing chronic inflammation-associated oral cancer as well as in chemoprevention.

\section{Acknowledgements}

The present study was supported by Dental Research Institute (grant no. PNUDH DRI-2015-02), Pusan National University Dental Hospital.

\section{References}

1. Katto J and Mahlknecht U: Epigenetic regulation of cellular adhesion in cancer. Carcinogenesis 32: 1414-1418, 2011. 
2. Liu J, Lin PC and Zhou BP: Inflammation fuels tumor progress and metastasis. Curr Pharm Des 21: 3032-3040, 2015.

3. Zhang L, Song X, Mohri Y and Qiao L: Role of inflammation and tumor microenvironment in the development of gastrointestinal cancers: What induced pluripotent stem cells can do? Curr Stem Cell Res Ther 10: 245-250, 2015.

4. Li Y, Wang L, Pappan L, Galliher-Beckley A and Shi J: IL-1 $\beta$ promotes stemness and invasiveness of colon cancer cells through Zeb1 activation. Mol Cancer 11: 87, 2012.

5. Diakos CI, Charles KA, McMillan DC and Clarke SJ: Cancer-related inflammation and treatment effectiveness. Lancet Oncol 15: e493-e503, 2014.

6. Bharti R, Dey G and Mandal M: Cancer development, chemoresistance, epithelial to mesenchymal transition and stem cells: A snapshot of IL-6 mediated involvement. Cancer Lett 375: 51-61, 2016.

7. Wang D, Fu L, Sun H, Guo L and DuBois RN: Prostaglandin E2 promotes colorectal cancer stem cell expansion and metastasis in mice. Gastroenterology 149: 1884-1895, 2015.

8. St John MA: Inflammatory mediators drive metastasis and drug resistance in head and neck squamous cell carcinoma. Laryngoscope 125 (Suppl 3): S1-S11, 2015.

9. Yadav A, Kumar B, Datta J, Teknos TN and Kumar P: IL-6 promotes head and neck tumor metastasis by inducing epithelial-mesenchymal transition via the JAK-STAT3-SNAIL signaling pathway. Mol Cancer Res 9: 1658-1667, 2011.

10. Eke PI, Dye BA, Wei L, Thornton-Evans GO and Genco RJ; CDC periodontal disease surveillance workgroup: James beck (university of north carolina, chapel hill, usa), gordon douglass (past president, american academy of periodontology), roy page (university of washin: Prevalence of periodontitis in adults in the united states: 2009 and 2010. J Dent Res 91: 914-920, 2012.

11. Natarajan E and Eisenberg E: Contemporary concepts in the diagnosis of oral cancer and precancer. Dent Clin North Am 55: 63-88, 2011.

12. Dehai C, Bo P, Qiang T, Lihua S, Fang L, Shi J, Jingyan C, Yan Y, Guangbin W and Zhenjun Y: Enhanced invasion of lung adenocarcinoma cells after co-culture with THP-1-derived macrophages via the induction of EMT by IL-6. Immunol Lett 160: 1-10, 2014

13. Kim S, Lee J, Jeon M, Lee JE and Nam SJ: MEK-dependent IL-8 induction regulates the invasiveness of triple-negative breast cancer cells. Tumour Biol 37: 4991-4999, 2016

14. Wang Y, Li L, Guo X, Jin X, Sun W, Zhang X and Xu RC: Interleukin-6 signaling regulates anchorage-independent growth, proliferation, adhesion and invasion in human ovarian cancer cells. Cytokine 59: 228-236, 2012.

15. Abiko Y, Sato T, Mayanagi G and Takahashi N: Profiling of subgingival plaque biofilm microflora from periodontally healthy subjects and from subjects with periodontitis using quantitative real-time PCR. J Periodontal Res 45: 389-395, 2010.

16. Wolff L, Dahlen G and Aeppli D: Bacteria as risk markers for periodontitis. J Periodontol 65: 498-510, 1994.

17. Chen X, Yang L, Oppenheim JJ and Howard MZ: Cellular pharmacology studies of shikonin derivatives. Phytother Res 16 : 199-209, 2002

18. Kim DJ, Lee JH, Park HR and Choi YW: Acetylshikonin inhibits growth of oral squamous cell carcinoma by inducing apoptosis. Arch Oral Biol 70: 149-157, 2016.

19. Lee HJ, Lee HJ, Magesh V, Nam D, Lee EO, Ahn KS, Jung MH Ahn KS, Kim DK, Kim JY and Kim SH: Shikonin, acetylshikonin and isobutyroylshikonin inhibit VEGF-induced angiogenesis and suppress tumor growth in lewis lung carcinoma-bearing mice. Yakugaku Zasshi 128: 1681-1688, 2008.

20. Moon J, Koh SS, Malilas W, Cho IR, Kaewpiboon C, Kaowinn S, Lee K, Jhun BH, Choi YW and Chung YH: Acetylshikonin induces apoptosis of hepatitis B virus $\mathrm{X}$ protein-expressing human hepatocellular carcinoma cells via endoplasmic reticulum stress. Eur J Pharmacol 735: 132-140, 2014

21. Ha NH, Woo BH, Kim DJ, Ha ES, Choi JI, Kim SJ, Park BS Lee JH and Park HR: Prolonged and repetitive exposure to porphyromonas gingivalis increases aggressiveness of oral cancer cells by promoting acquisition of cancer stem cell properties. Tumour Biol 36: 9947-9960, 2015.

22. Okamoto A, Chikamatsu K, Sakakura K, Hatsushika K, Takahashi G and Masuyama K: Expansion and characterization of cancer stem-like cells in squamous cell carcinoma of the head and neck. Oral Oncol 45: 633-639, 2009.
23. Cao L, Zhou Y, Zhai B, Liao J, Xu W, Zhang R, Li J, Zhang Y, Chen L, Qian H, et al: Sphere-forming cell subpopulations with cancer stem cell properties in human hepatoma cell lines. BMC Gastroenterol 11: 71, 2011.

24. Murray GI, Duncan ME, O'Neil P, Melvin WT and Fothergill JE: Matrix metalloproteinase-1 is associated with poor prognosis in colorectal cancer. Nat Med 2: 461-462, 1996.

25. Gu ZD, Li JY, Li M, Gu J, Shi XT, Ke Y and Chen KN: Matrix metalloproteinases expression correlates with survival in patients with esophageal squamous cell carcinoma. Am J Gastroenterol 100: 1835-1843, 2005.

26. Gong L, Wu D, Zou J, Chen J, Chen L, Chen Y, Ni C and Yuan H: Prognostic impact of serum and tissue MMP-9 in non-small cell lung cancer: A systematic review and meta-analysis. Oncotarget 7: 18458-18468, 2016.

27. Bodet C, Chandad F and Grenier D: Modulation of cytokine production by porphyromonas gingivalis in a macrophage and epithelial cell co-culture model. Microbes Infect 7: 448-456, 2005.

28. Sandros J, Karlsson C, Lappin DF, Madianos PN, Kinane DF and Papapanou PN: Cytokine responses of oral epithelial cells to porphyromonas gingivalis infection. J Dent Res 79: 1808-1814, 2000.

29. Zhao JJ, Feng XP, Zhang XL and Le KY: Effect of porphyromonas gingivalis and lactobacillus acidophilus on secretion of IL1B, IL6 and IL8 by gingival epithelial cells. Inflammation 35: 1330-1337, 2012.

30. Beklen A, Ainola M, Hukkanen M, Gürgan C, Sorsa T and Konttinen YT: MMPs, IL-1 and TNF are regulated by IL-17 in periodontitis. J Dent Res 86: 347-351, 2007.

31. Franco C, Patricia HR, Timo S, Claudia B and Marcela H: Matrix metalloproteinases as regulators of periodontal inflammation. Int J Mol Sci 18: E440, 2017.

32. Mauviel A: Cytokine regulation of metalloproteinase gene expression. J Cell Biochem 53: 288-295, 1993.

33. Teng J, Wang X, Xu Z and Tang N: HBx-dependent activation of twist mediates STAT3 control of epithelium-mesenchymal transition of liver cells. J Cell Biochem 114: 1097-1104, 2013.

34. Iqbal J, McRae S, Banaudha K, Mai T and Waris G: Mechanism of hepatitis $\mathrm{C}$ virus (HCV)-induced osteopontin and its role in epithelial to mesenchymal transition of hepatocytes. J Biol Chem 288: 36994-37009, 2013.

35. Horikawa T, Yoshizaki T, Kondo S, Furukawa M, Kaizaki Y and Pagano JS: Epstein-barr virus latent membrane protein 1 induces snail and epithelial-mesenchymal transition in metastatic nasopharyngeal carcinoma. Br J Cancer 104: 1160-1167, 2011.

36. Chandrakesan P, Roy B, Jakkula LU, Ahmed I, Ramamoorthy P, Tawfik O, Papineni R, Houchen C, Anant S and Umar S: Utility of a bacterial infection model to study epithelial-mesenchymal transition, mesenchymal-epithelial transition or tumorigenesis. Oncogene 33: 2639-2654, 2014.

37. Sztukowska MN, Ojo A, Ahmed S, Carenbauer AL, Wang Q, Shumway B, Jenkinson HF, Wang H, Darling DS and Lamont RJ: Porphyromonas gingivalis initiates a mesenchymal-like transition through ZEB1 in gingival epithelial cells. Cell Microbiol 18: 844-858, 2016

38. Ha NH, Park DG, Woo BH, Kim DJ, Choi JI, Park BS, Kim YD, Lee JH and Park HR: Porphyromonas gingivalis increases the invasiveness of oral cancer cells by upregulating IL- 8 and MMPs. Cytokine 86: 64-72, 2016.

39. Wang Y, Xu RC, Zhang XL, Niu XL, Qu Y, Li LZ and Meng XY: Interleukin-8 secretion by ovarian cancer cells increases anchorage-independent growth, proliferation, angiogenic potential, adhesion and invasion. Cytokine 59: 145-155, 2012.

40. Ou Z, Wang Y, Liu L, Li L, Yeh S, Qi L and Chang C: Tumor microenvironment $\mathrm{B}$ cells increase bladder cancer metastasis via modulation of the IL-8/androgen receptor (AR)/MMPs signals. Oncotarget 6: 26065-26078, 2015.

41. George VC, Dellaire G and Rupasinghe HPV: Plant flavonoids in cancer chemoprevention: Role in genome stability. J Nutr Biochem 45: 1-14, 2017

42. Niedzwiecki A, Roomi MW, Kalinovsky T and Rath M: Anticancer efficacy of polyphenols and their combinations. Nutrients 8: E552, 2016

This work is licensed under a Creative Commons Attribution-NonCommercial-NoDerivatives 4.0 International (CC BY-NC-ND 4.0) License. 